\title{
Down type isosinglet quarks in ATLAS
}

\author{
R. Mehdiyev ${ }^{1,2}$, A. Siodmok ${ }^{3,4}$, S. Sultansoy ${ }^{2,5}$, G. Unel ${ }^{4,6, a}$ \\ ${ }^{1}$ Université de Montréal, Département de Physique, Montréal, Canada \\ ${ }^{2}$ Institute of Physics, Academy of Sciences, Baku, Azerbaijan \\ 3 Jagiellonian University, Physics Department, Cracow, Poland \\ ${ }^{4}$ CERN, Physics Department, 1211 Geneva, Switzerland \\ 5 TOBB University of Economics and Technology, Physics Department, Ankara, Turkey \\ ${ }^{6}$ University of California at Irvine, Physics Department, Irvine, CA 92697, USA
}

Received: 19 November 2007 / Revised version: 22 January 2008 /

Published online: 16 February 2008 - (C) Springer-Verlag / Società Italiana di Fisica 2008

\begin{abstract}
We evaluate the discovery reach of the ATLAS experiment for down type isosinglet quarks, $D$, using both their neutral and charged decay channels, namely the process $p p \rightarrow D \bar{D}+X$ with subsequent decays resulting in $2 \ell+2 j+E_{T}, 3 \ell+2 j+E_{T}$ and $2 \ell+4 j$ final states. The integrated luminosity required for observation of a heavy quark is estimated for a mass range between 600 and $1000 \mathrm{GeV}$ using the combination of results from different search channels.
\end{abstract}

\section{Introduction}

In an effort to unify the forces known to the standard model (SM) within a grand unified theory (GUT), one is led to consider extended families of fermions. One of the often quoted GUT symmetry groups having $\mathrm{SU}_{C}(3) \times$ $\mathrm{SU}_{W}(2) \times \mathrm{U}_{Y}(1)$ of the $\mathrm{SM}$ as a subgroup is the exceptional Lie group $E_{6}$ with its 27 dimensional fundamental representation [1-3]. Such a large symmetry group not only puts the known quarks and leptons in the same family, but also predicts additional particles, such as isosinglet quarks and new heavy gauge bosons. In the literature, the new charge $q=-1 / 3$ isosinglet quarks are denoted by the letters $D, S$, and $B$, representing the additions to the first, second and third SM families, respectively.

Here, we investigate the discovery potential of the ATLAS experiment for the down type isosinglet quark of the first SM family ( $D$ quark) at the forthcoming LHC accelerator. In accordance with the observed quark mass hierarchy, we will assume the first family's isosinglet quark $D$ to be the lightest of the particles predicted by the $E_{6}$ group and hence the first one accessible at the LHC energy. It is assumed that the other new particles predicted by the model, being very massive, do not contribute to the $D$ quark search. The present experimental limit on the mass of such an isosinglet quark is $m_{D}>199 \mathrm{GeV}$ [4]. A second assumption will be that the intra-family mixing is stronger than the mixing between families. Although we will concentrate only on the $D$ quark, the results will stay valid also for the $S$ quark, as jets from first and second quark generations are experimentally indistinguishable. The investigation for the $B$ quark requires detailed information

\footnotetext{
a e-mail: gokhan.unel@cern.ch
}

about the detector response to $b$-jets which is beyond the scope of this work.

The Lagrangian relevant for the weak interactions of $d$ and $D$ quarks is given as [5] :

$$
\begin{aligned}
L_{D}= & \frac{\sqrt{4 \pi \alpha_{\mathrm{em}}}}{2 \sqrt{2} \sin \theta_{\mathrm{W}}}\left[\bar{u}^{\theta} \gamma_{\alpha}\left(1-\gamma_{5}\right) d \cos \phi\right. \\
& \left.+\bar{u}^{\theta} \gamma_{\alpha}\left(1-\gamma_{5}\right) D \sin \phi\right] W^{\alpha} \\
& -\frac{\sqrt{4 \pi \alpha_{\mathrm{em}}}}{4 \sin \theta_{\mathrm{W}}}\left[\frac{\sin \phi \cos \phi}{\cos \theta_{\mathrm{W}}} \bar{d} \gamma_{\alpha}\left(1-\gamma_{5}\right) D\right] Z^{\alpha} \\
& -\frac{\sqrt{4 \pi \alpha_{\mathrm{em}}}}{12 \cos \theta_{\mathrm{W}} \sin \theta_{\mathrm{W}}} \\
& \times\left[\bar{D} \gamma_{\alpha}\left(4 \sin ^{2} \theta_{\mathrm{W}}-3 \sin ^{2} \phi\left(1-\gamma_{5}\right)\right) D\right. \\
& \left.+\bar{d} \gamma_{\alpha}\left(4 \sin ^{2} \theta_{\mathrm{W}}-3 \cos ^{2} \phi\left(1-\gamma_{5}\right)\right) d\right] Z^{\alpha}+\text { h.c. },
\end{aligned}
$$

where the superscript $\theta$ represents the usual CKM mixings taken to be in the up sector for simplicity of calculation, $\theta_{\mathrm{W}}$ is the weak angle and $\phi$ is the mixing angle between the $d$ and $D$ quarks. This mixing is responsible for the decay of the $D$ quark. The limits on $\phi$ can be obtained from the current precision measurements for the $3 \times 3$ CKM matrix elements assuming that its $3 \times 4$ extension has the sum of the squares of the elements of a row equal to one. The evaluation of the present [4] values yield $|\sin \phi| \leq 0.045$. Although the upper limit is used throughout this work, the results are essentially insensitive to $\sin \phi$ as the pair production considered here proceeds mostly via gluon fusion.

The SM fermions acquire their masses through their interactions with the isodoublet Higgs field. The Higgs mechanism can also be preserved in the $E_{6}$ group structure as an effective theory, although other alternatives such as dy- 
namical symmetry breaking are also proposed $[6,7]$. On the other hand, the origin of the mass of the new quarks $(D$, $S, B$ ) should be due to another mechanism since they are isosinglets. For this reason and following the literature [3], (1) does not contain Higgs related terms. However, if the mass of the Higgs boson is smaller than the isosinglet quark mass and if the interaction between the Higgs field and $d$ quark is considered before the spontaneous symmetry breaking but after $d-D$ quark mixing, a new $D$ quark decay channel becomes available: $D \rightarrow h d$. The effect of such interaction, depending on the Higgs boson and $D$ quark masses, is to reduce the $\operatorname{BR}(D \rightarrow W u)$ from $66 \%$ down to $50 \%$ and $\mathrm{BR}(D \rightarrow Z d)$ from $33 \%$ down to $25 \%$, giving way to $\operatorname{BR}(D \rightarrow h d)=25 \%$ (for details see [8] and [9]).

\section{Analysis}

The leading Feynman diagrams for the pair production of the $D$ quark at the LHC are given in Fig. 1. The Lagrangian in (1) was implemented in two tree level generators: CompHep [10,11] and MadGraph [12]. The pair production signal cross section was calculated in both generators as a function of $D$ quark mass, yielding practically equal cross sections for the same generator level cuts. The result from CompHep is plotted as a function of the new quark mass in Fig. 2. Contributions from the electroweak interactions to the production cross section are found to be much smaller than those from the strong interactions. Below a $D$ quark mass of about $700 \mathrm{GeV}$, the dominant contribution to the production cross section is from the



gluons, s channel

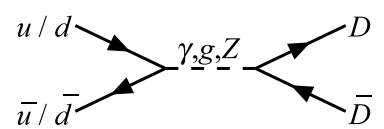

up quarks, s channel

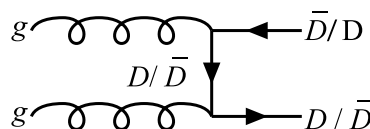

gluons, t channel

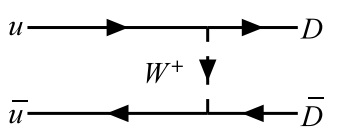

up quarks, $\mathrm{t}$ channel
Fig. 1. Main diagrams for the pair production of the $D$ quark at the LHC diagrams in the top row of Fig. 2 but as the mass increases, the quark fusion contribution takes over according to CTEQ6L1 PDFs [13]. One should note that single production of $D$ quarks (in association with a light SM quark) is also possible in this model. However, since this type of subprocesses, in comparison to the pair production, would give a different final state the production of single $D$ quarks is considered in a separate study [14].

The $E_{6}$ GUT model does not predict the masses of the isosinglet quarks. Therefore, this study scans some plausible values for the D quark mass $(600-1000 \mathrm{GeV})$ to estimate the experimental reach of the ATLAS detector [15]. With such a large mass, the isosinglet quarks are expected to immediately decay into SM particles. A number of decay channels of the $D$ quark pairs are summarized in Table 1 . The process in the first row has been previously studied and found to be feasible for discovery up to a mass of about $1 \mathrm{TeV}$, for an integrated luminosity of $200 \mathrm{fb}^{-1}[16]$. The present work aims to evaluate the possibility of discovery of the $D$ quark in the production channels shown in the remaining rows of the same table using the final states in column 3 . The high transverse momentum of the jets com-

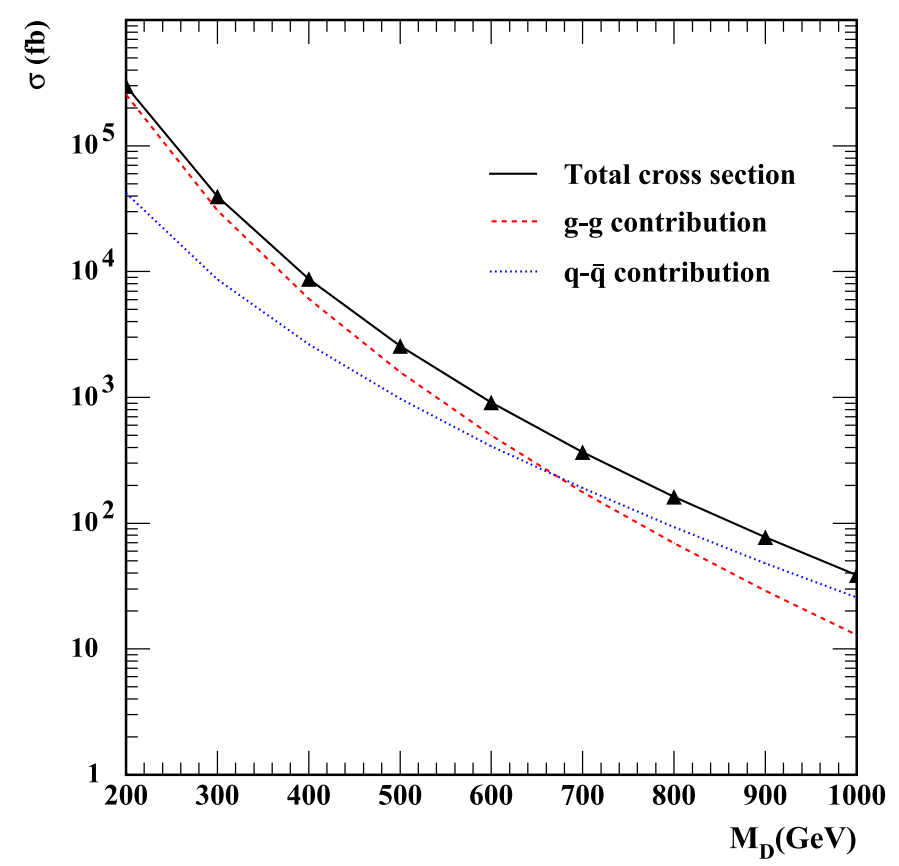

Fig. 2. Tree level pair production of $D$ quarks at LHC as a function of the new quark's mass

Table 1. List of the studied signal channels: the last column contains the total branching ratios to the final state particles where $\ell$ represents $\mu$ or $e$

\begin{tabular}{|c|c|c|c|c|c|}
\hline \multirow{2}{*}{$\frac{\overline{D \bar{D} \rightarrow}}{Z Z d \bar{d}}$} & \multicolumn{2}{|c|}{ Final state } & \multirow{2}{*}{$\frac{\text { Expected signal }}{4 \ell+2 \text { jet }}$} & \multirow{2}{*}{$\begin{array}{c}\text { Decay B.R. } \\
0.07 \times 0.07\end{array}$} & \multirow{2}{*}{$\begin{array}{c}\text { Total B.R. } \\
0.0005\end{array}$} \\
\hline & $Z \rightarrow \ell \bar{\ell}$ & $Z \rightarrow \ell \bar{\ell}$ & & & \\
\hline $0.33 \times 0.33$ & $Z \rightarrow \ell \bar{\ell}$ & $Z \rightarrow \nu \bar{\nu}$ & $2 \ell+2$ jet $+E_{T}$ & $2 \times 0.07 \times 0.2$ & 0.0028 \\
\hline & $Z \rightarrow \ell \bar{\ell}$ & $Z \rightarrow q \bar{q}$ & $2 \ell+4$ jet & $2 \times 0.07 \times 0.7$ & 0.0107 \\
\hline$Z W d u$ & $Z \rightarrow \ell \bar{\ell}$ & $W \rightarrow l \bar{\nu}$ & $3 \ell+2 \mathrm{jet}+E_{T}$ & $0.07 \times 0.21$ & 0.0065 \\
\hline $2 \times 0.66 \times 0.33$ & $Z \rightarrow \ell \bar{\ell}$ & $W \rightarrow q \bar{q}$ & $2 \ell+4$ jet & $0.07 \times 0.68$ & 0.0211 \\
\hline
\end{tabular}


ing from the $D$ quark decays can be used to identify the signal events. As for the backgrounds, all the SM tree level processes yielding two jets and two vector bosons $(Z-Z$ and $W-Z$ ) are considered. In this feasibility study, the detector related background events originating nor from jet combinatorics, neither from the lepton and jet misidentifications such as two vector bosons plus one or three jets, are taken into account. The background cross sections were calculated only in MadGraph as this generator is suitable to be used on large computing farms. In the numerical calculations, we set the QCD scale of the signal to the mass of the new quark while keeping it as the mass of the $Z$ boson for the background, yielding more conservative results compared to the investigation in [16]. For example, the $3 \sigma$ observation limit gets reduced by $15 \%$ in the $4 \ell+2 j$ channel studied in that work. The simple requirements imposed at the generator level are:

$$
\begin{aligned}
\left|\eta_{p}\right| & <2.5, \\
p_{\mathrm{T}, p} & >10 \mathrm{GeV}, \\
R_{p \bar{p}} & >0.4 \\
\left|\eta_{Z, W}\right| & <5.0
\end{aligned}
$$

where $R$ is the cone separation angle between two leading partons $(p)$ giving rise to two jets. Selection of the pseudorapidity region is driven by partonic spectra distributions, which are peaked in the central region. Decays of $Z$ and $W$ bosons were performed by PYTHIA [17] which handled the initial and final state radiation together with the hadronization. The detector response was estimated using the ATLAS fast simulation program ATLFAST [18] within the offline analysis framework, ATHENA v11.0.41 .

\subsection{Search using the $2 \ell+2 j+Z_{T}$ channel}

This search aims to exploit the relatively large branching ratio seen in second line of Table 1 . However the invisible decay of one $Z$ boson, makes reconstruction of both $D$ quarks impossible. The expected viable signature is two isolated leptons, two energetic jets and large missing transverse energy. The reconstructed invariant mass from the two leptons is required to yield the $Z$ boson mass with an error of $\pm 20 \mathrm{GeV}$ which is an order of magnitude larger than the reconstruction resolution $\pm 2.5 \mathrm{GeV}$. The two most energetic jets are selected as candidates for the parton daughters of the $D$ quark decays. However, the ambiguity in the association of a jet and a $Z$ boson, makes each event contribute twice to the $D$ quark invariant mass histogram, one with the correct association and one with the wrong one. The background for these events is estimated by taking into account all the SM processes which give two energetic jets and two $Z$ bosons, allowing one of them to decay invisibly and the other one into electrons or muons. The total cross section of such processes with the generator level cuts in (2) except $p_{\mathrm{T}, p}>100 \mathrm{GeV}$ is $2.65 \mathrm{pb}$. The other SM processes with $2 \ell+$ $2 j+E_{T}$ background are expected to be negligible, given the high $E_{T}$ in the topology of the signal events, and the $Z$ boson mass reconstruction requirement.
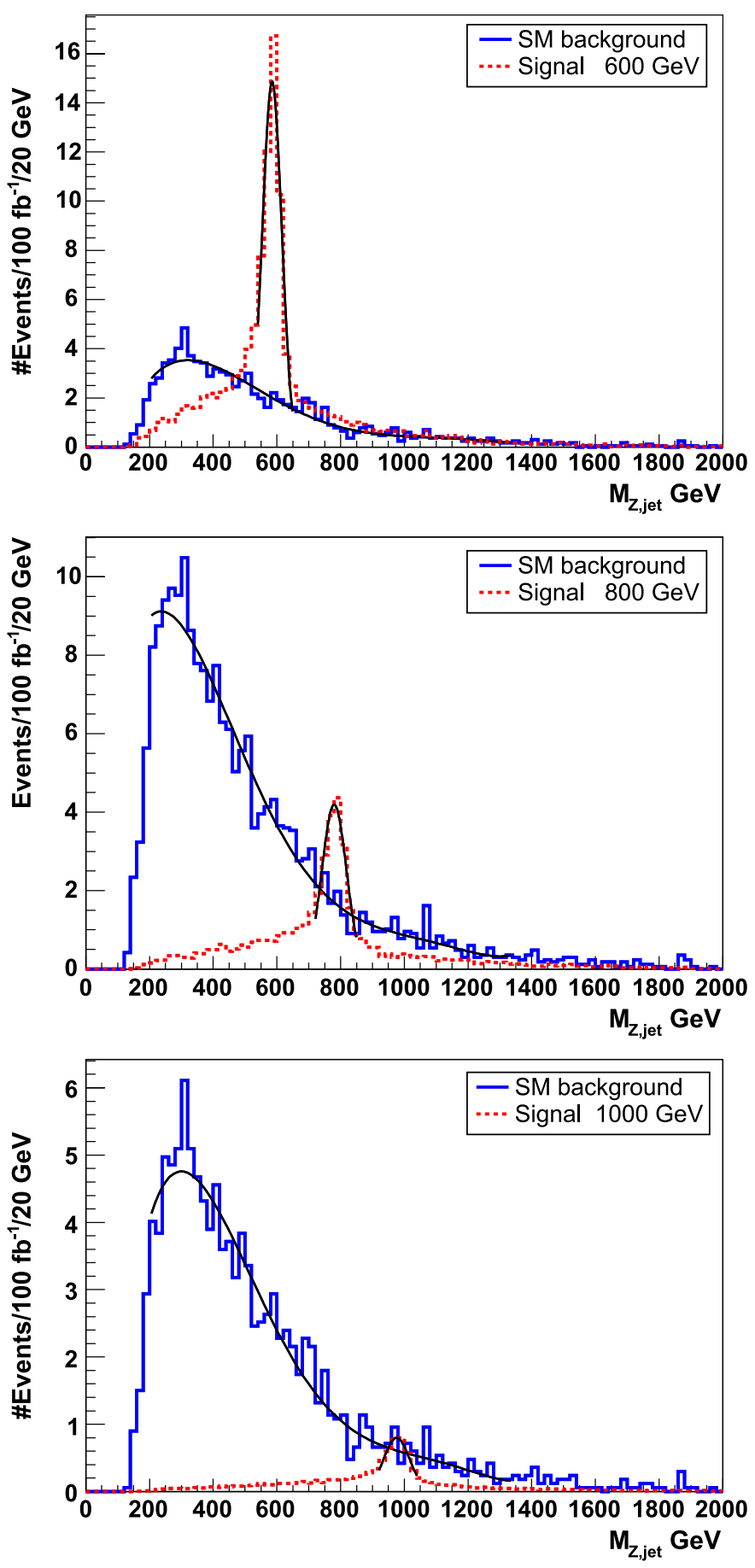

Fig. 3. Invariant mass spectra of signal and background for different values of $D$ quark mass in $2 \ell+2 j+E_{T}$ channel

The simple selection criteria for this channel for a $D$ quark candidate of $600 \mathrm{GeV}$ are presented in the set below:

$$
\begin{aligned}
\#(e, \mu) & =2, \\
\#_{T} & \geq 150 \mathrm{GeV}, \\
M_{\ell \bar{\ell}} & =90 \pm 20 \mathrm{GeV}, \\
\# \text { jets } & \geq 2, \\
p_{\mathrm{T}} \mathrm{jet} & \geq 150 \mathrm{GeV} .
\end{aligned}
$$


Table 2. The signal and background cross sections before and after the event selection (B.S. and A.S.) with the relevant branching ratios folded in. The expected number of signal $(S)$ and background $(B)$ events in the range of $\pm 2 \sigma$ from the center of the Gaussian fit to the peak and the statistical significance for $2 \ell+2 j+Z_{T}$ channel after the event selection criteria in equation set (3) for an integrated luminosity of $100 \mathrm{fb}^{-1}$ is also given

\begin{tabular}{lccc}
\hline Mass $(\mathrm{GeV})$ & 600 & 800 & 1000 \\
\hline$\sigma_{\text {signal }}$ B.S. (fb) & 3.11 & 0.59 & 0.12 \\
$\sigma_{\text {signal }}$ A.S. (fb) & 1.17 & 0.43 & 0.097 \\
$\sigma_{Z Z j j}$ B.S. (fb) & 37.9 & 37.9 & 37.9 \\
$\sigma_{Z Z j j}$ A.S. (fb) & 0.94 & 2.06 & 1.21 \\
$S$ & 53 & 19 & 4 \\
$B$ & 12 & 13 & 5 \\
significance & 15.3 & 5.3 & 2.1 \\
\hline
\end{tabular}

The resulting invariant mass plots for different $D$ quark mass values, with cut optimizations in each case, are given in Fig. 3 for one year of LHC data taking which corresponds to an integrated luminosity of $100 \mathrm{fb}^{-1}$. The solid histograms represent the events originating from the SM background and the dashed ones from the signal. Both distributions were fitted separately to reduce statistical fluctuations and to better estimate the total number of events in the histograms. The number of events for both signal and background were computed by integrating the fit functions in the range of $\pm 2 \sigma$ from the center of the Gaussian fit to the signal peak. Table 2 contains the number of signal $(S)$ and background $(B)$ events together with the signal significance calculated in this way for an integrated luminosity of $100 \mathrm{fb}^{-1}$. In this note, the signal significance is calculated as $S / \sqrt{B}$ if $S+B>25$, otherwise Poisson statistics are used to obtain the probability $P$ of compatibility with the background. In the latter case the significance of the Gaussian distribution yielding the same probability, $P$, is given.

\subsection{Search using the $3 \ell+2 j+\mathbb{E}_{T}$ channel}

This channel has one $D$ quark decaying through a $Z$ boson and another $D$ quark decaying via a $W$ boson, i.e. $D D \rightarrow Z W j j$ final state. The $Z$ boson is expected to be entirely reconstructed from two electrons or two muons. The $W$ boson can also be partially reconstructed if a good measurement of missing $E_{\mathrm{T}}$ can be achieved. The signal is sought in the final state made of a pair of energetic leptons (electrons or muons), accompanied by a third high $p_{\mathrm{T}}$ lepton (muon or electron), two high $p_{\mathrm{T}}$ jets and the missing transverse energy.

For the background studies, events consisting of the SM $W Z+2 j$ processes were generated. The background cross section before event selection is $2.30 \mathrm{pb}$ from the $W^{-}$case and $3.89 \mathrm{pb}$ from $W^{+}$case, summing up to $6.19 \mathrm{pb}$ with the generator level cuts in (2) except $p_{\mathrm{T}, p}>50 \mathrm{GeV}$. The composition of background final state and allowed decay modes of $Z$ and $W$ bosons were chosen to be exactly the same as for the signal events. The other possible background contributions due to misidentified or undetected leptons, such as $Z Z+2 j$ or $W W+2 j$ events, will be significantly suppressed due to the event selection: rather high transverse momentum cuts on jets and the require-


Fig. 4. Jet (left), electron (center) and muon (right) transverse momentum spectra for $3 \ell+2 j+E_{T}$ channel. The shaded area represents the SM background contribution 

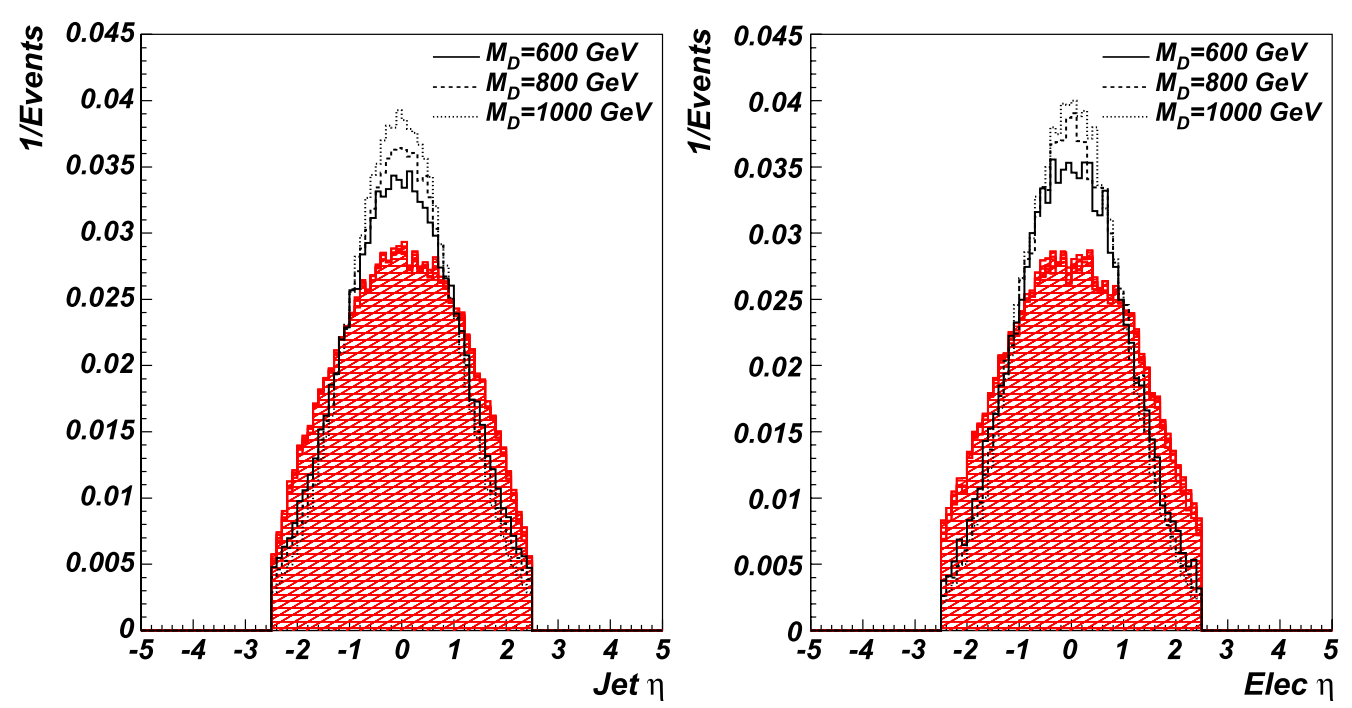

Fig. 5. Jet (left) and electron (right) pseudorapidity distributions for different $D$ quark masses using the $3 \ell+2 j+Z_{T}$ channel. The shaded area represents the SM background contribution

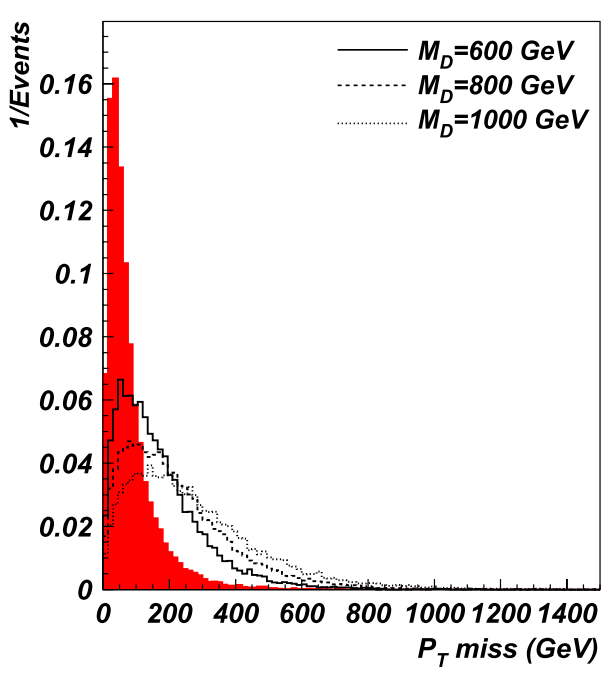

Fig. 6. Missing $p_{\mathrm{T}}$ distributions for different $D$ quark masses using the $3 \ell+2 j+Z_{T}$ channel. The shaded area represents the SM background contribution

ment of reconstructing both $Z$ and $W$ bosons in the same event.

Two cases were separately considered during the event selection for the lepton composition of the final state. These two cases and the event selection cuts for a $D$ quark mass of $600 \mathrm{GeV}$ are:

A. the $Z$ and $W$ decays involving leptons of the same generation (electrons or muons) were selected using

$$
\# e=3 \text { or } \# \mu=3 \text {; }
$$

B. the $Z$ and $W$ decays involving leptons of different generation (electrons or muons) were selected using

$$
\# e=2 \quad \# \mu=1 \quad \text { or } \quad \# e=1 \quad \# \mu=2 .
$$

The second case aims to ease the identification of leptons originating from $Z$ and $W$ bosons. The other analysis

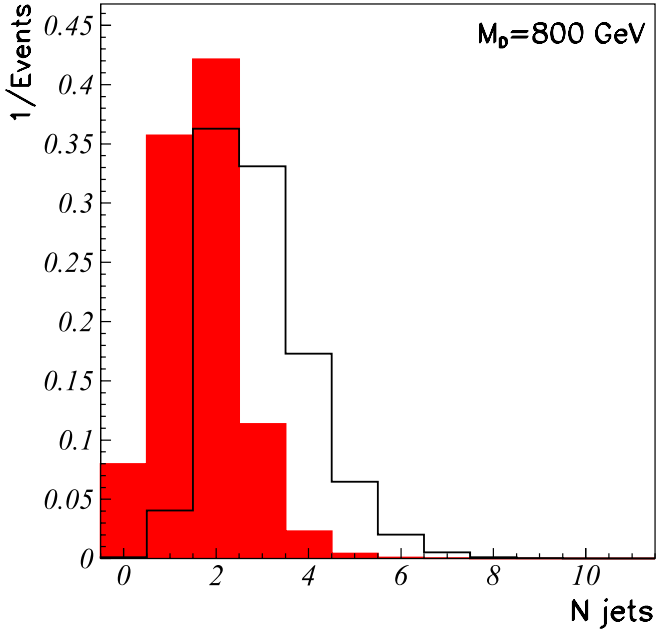

Fig. 7. Number of jets with $p_{\mathrm{T}}>70 \mathrm{GeV}$ for signal (white) and background (shaded) events in the $3 \ell+2 j+\mathbb{F}_{T}$ channel for an example $D$ quark mass of $800 \mathrm{GeV}$

cuts are common to both cases:

$$
\begin{aligned}
p_{\mathrm{T}, j} & >80 \mathrm{GeV}, \\
p_{\mathrm{T}, e, \mu} & >20 \mathrm{GeV}, \\
\mid \eta(j, e \text { or } \mu) \mid & <2.5, \\
p_{\mathrm{T}}^{\mathrm{miss}} & >30 \mathrm{GeV}, \\
M_{\ell \ell}^{\mathrm{rec}} & =90 \pm 20 \mathrm{GeV}, \\
M_{\ell \nu}^{\mathrm{rec}} & =80 \pm 20 \mathrm{GeV} .
\end{aligned}
$$

For higher $D$ quark mass values, the jet transverse momentum cuts were slightly increased to obtain a favorable significance value wherever it was statistically possible in both cases.

Transverse momentum spectra of jets, electrons and muons in the signal events for various $D$ quark mass in comparison with the corresponding spectra from the background events, are presented in Fig. 4 after the selection cuts defined above. One can see that the signal events fea- 

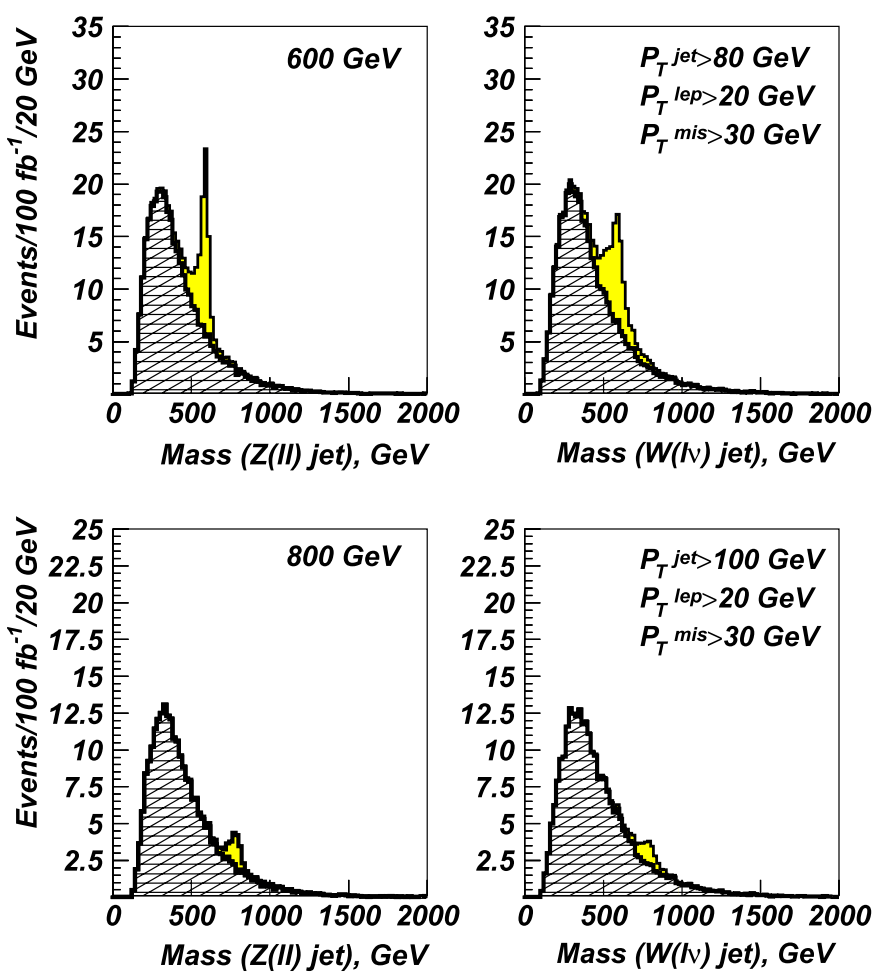

Fig. 8. Invariant mass reconstruction spectra of signal (shaded) and background (hashed) for two values of $D$ quark in $3 \ell+$ $2 j+E_{T}$ channel. The same lepton flavor was found in $Z$ and $W$ decay chains (Case A)

Table 3. The signal and background cross sections before and after the event selection (B.S. and A.S.) with the leptonic branching ratios folded in. The expected number of signal $(S)$ and background $(B)$ events in mass windows of width $\delta M$ around the peak and the statistical significance for the $3 \ell+2 j+E_{T}$ channel, after the cuts in equation set (6), is also given for type A (same lepton generation) using $100 \mathrm{fb}^{-1}$ of integrated luminosity

\begin{tabular}{lccc}
\hline Mass $(\mathrm{GeV})$ & 600 & 800 & 1000 \\
\hline$\sigma_{\text {signal }}$ B.S. (fb) & 3.2 & 0.6 & 0.11 \\
$\sigma_{\text {signal }}$ A.S. (fb) & 0.82 & 0.15 & 0.010 \\
$\sigma_{Z W j j}$ B.S. (fb) & 45.14 & 45.14 & 45.14 \\
$\sigma_{Z W j j}$ A.S. (fb) & 2.57 & 2.7 & 0.044 \\
$\delta M(D \rightarrow Z$ jet $)(\mathrm{GeV})$ & 120 & 140 & 160 \\
$\delta M(D \rightarrow W$ jet $)(\mathrm{GeV})$ & 200 & 220 & 240 \\
$S$ & 45 & 8.3 & 0.5 \\
$B$ & 11.4 & 4 & 0.25 \\
significance & 13.3 & 3.3 & - \\
\hline
\end{tabular}

ture more energetic jets and leptons than the background data. Pseudorapidity distributions of jets and electrons in the same events are shown on Fig. 5. Partons from signal events are produced mostly in the central region, more so as the $D$ quark mass increases. Missing transverse momentum distribution, produced in the event as a result of the semi-leptonic $W$ decay is shown on the Fig. 6 . As it can be seen, signal events produce more energetic $W$ bosons,
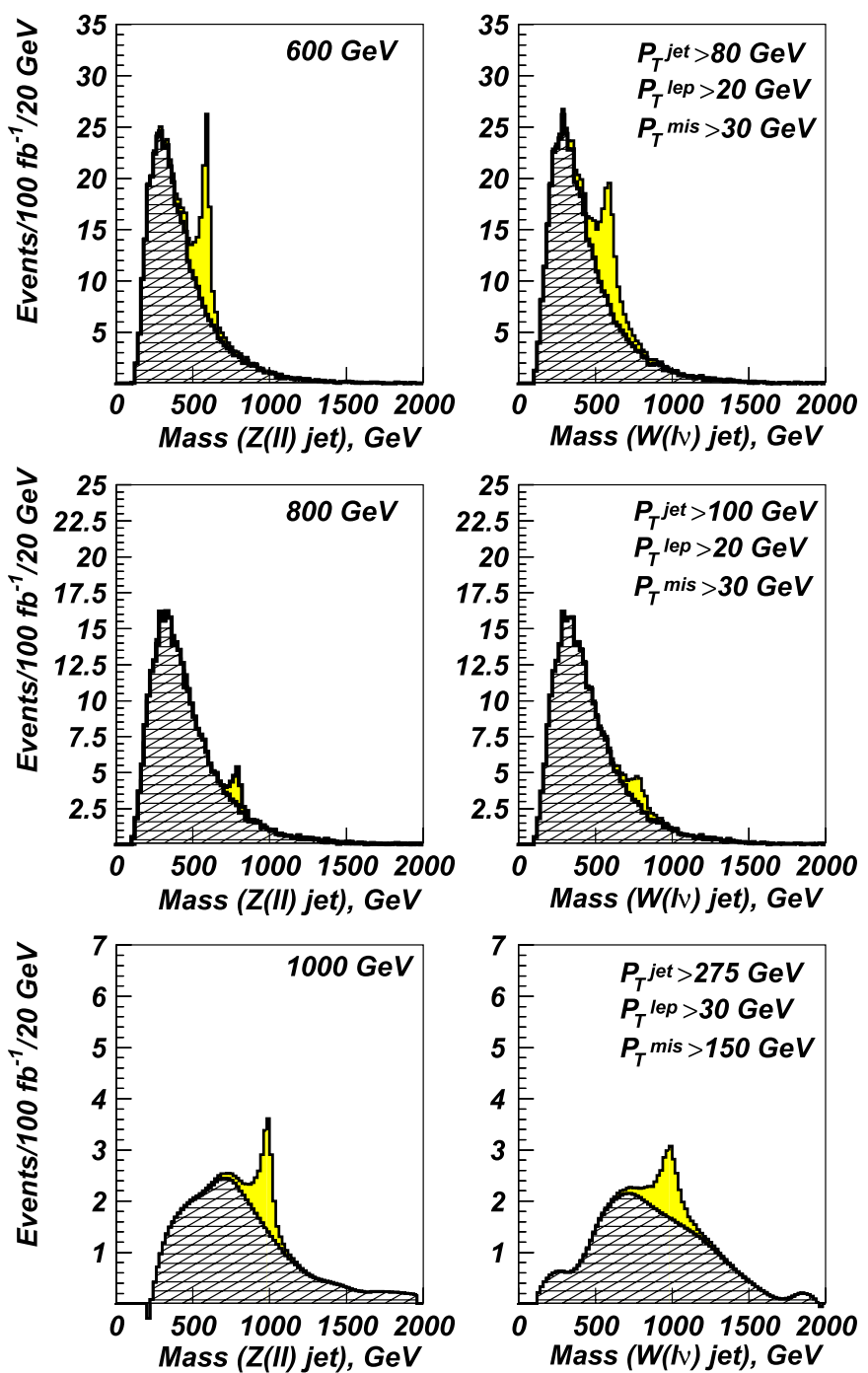

Fig. 9. Invariant mass reconstruction spectra of signal (shaded) and background (hashed) for three values of $D$ quark in $3 \ell+$ $2 j+E_{T}$ channel. The lepton flavor found in $Z$ and $W$ decay chains was different (case B)

which results in larger missing transverse momentum vector with respect to the $\mathrm{SM} W Z j j$ events.

The reconstructed invariant mass of the two leptons was required to be in the $(90 \pm 20) \mathrm{GeV}$ mass window to ensure their origin from the $Z$ boson. The $W$ boson "visible mass" was reconstructed from the measured lepton momenta and the $E_{T}$ in the transverse plane, assuming negligible neutrino longitudinal momentum. A mass window constraint of $(80 \pm 20) \mathrm{GeV}$ was applied to the reconstructed $M_{\ell \nu}^{\text {rec }}$ to select events with a $W$ boson.

In case $\mathrm{A}$, the identification of the two leptons originating from the $Z$ boson decay forced the use of the third lepton to reconstruct the $W$ boson invariant mass. The method used to identify these two leptons and thus to avoid the problems due combinatorics related to the issue of lepton assignment to $Z$ or $W$, was to select those giving the most accurate $Z$ boson mass. In case B, as the leptons originating from $Z$ boson were of a different SM family as 
Table 4. The signal and background cross sections before and after the event selection (B.S. and A.S.) with the leptonic decay branching ratios folded in. The expected number of signal $(S)$ and background $(B)$ events in the mass window around the peak and the statistical significance for the $3 \ell+2 j+Z_{T}$ channel, after the cuts given in equation set (6), for type B (different lepton generations) using $100 \mathrm{fb}^{-1}$ of data

\begin{tabular}{lccc}
\hline Mass $(\mathrm{GeV})$ & 600 & 800 & 1000 \\
\hline$\sigma_{\text {signal }}$ B.S. $(\mathrm{fb})$ & 3.2 & 0.6 & 0.11 \\
$\sigma_{\text {signal }}$ A.S. $(\mathrm{fb})$ & 1.0 & 0.2 & 0.014 \\
$\sigma_{Z W j j}$ B.S. $(\mathrm{fb})$ & 45.14 & 45.14 & 45.14 \\
$\sigma_{Z W j j}$ A.S. $(\mathrm{fb})$ & 4.8 & 3.4 & 0.1 \\
$\delta M(D \rightarrow Z$ jet$)(\mathrm{GeV})$ & 120 & 140 & 160 \\
$\delta M(D \rightarrow W$ jet$)(\mathrm{GeV})$ & 200 & 220 & 240 \\
$S$ & 52 & 10 & 1.3 \\
$B$ & 13.5 & 5 & 0.8 \\
significance & 14.2 & 3.8 & - \\
\hline
\end{tabular}

compared to the one from the $W$ decay, reconstruction of the $W$ invariant mass was straightforward.

As QCD radiation from energetic partons may result in an increased number of produced jets, a correct assignment of selected jets might not always be possible due to combinatorics. Moreover, the signal events produce a significant number of jets, as can be seen from Fig. 7, where the jet $\left(p_{\mathrm{T}}>70 \mathrm{GeV}\right)$ multiplicity distributions for the signal events of a $D$ quark mass $800 \mathrm{GeV}$ and for the background events are presented. Such a large jet multiplicity could pose a problem for the identification of the jet originating from the $D$ quark decay and hence for the reconstruction of its invariant mass. In both cases the invariant mass of the $D$ quark, decaying to a $Z$ boson and a jet was reconstructed by combining those two leptons with the most energetic (or the next to most energetic) jets only. The ambiguity in assigning the two most energetic jets to the two reconstructed bosons can be solved by calculating the invariant mass of the $D$ quarks using all possible combinations and by taking the one which gives the smallest mass difference between the $D$

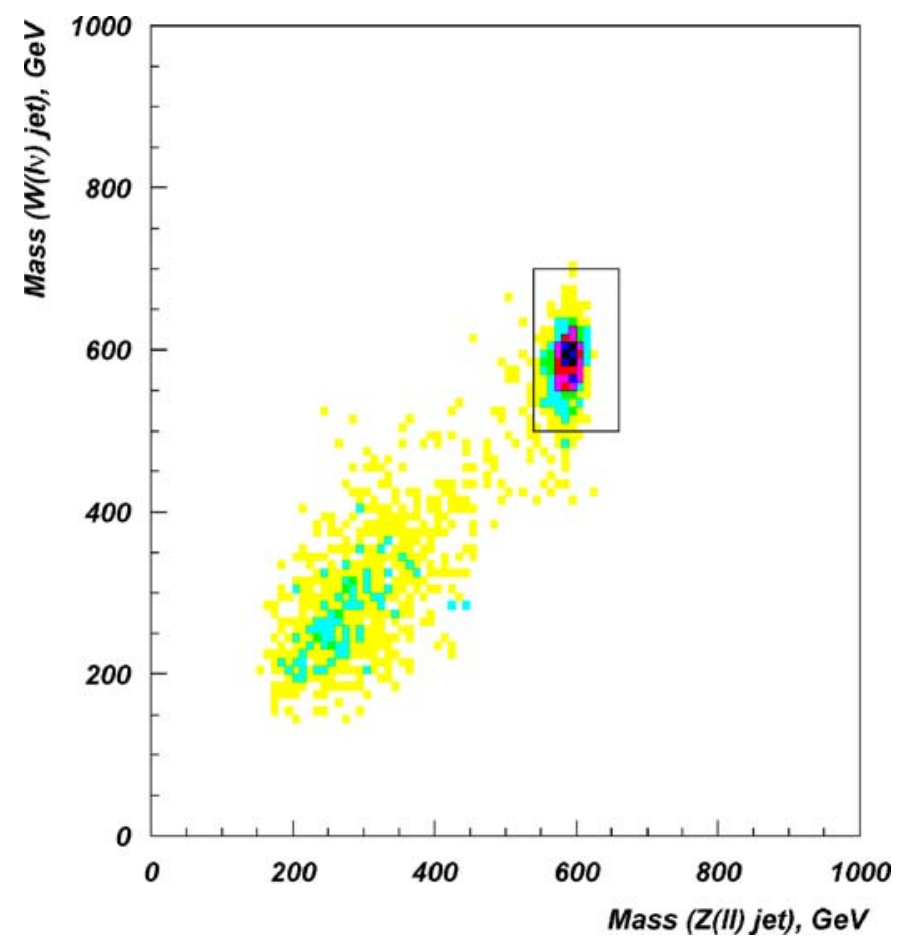

Fig. 10. Example of the rectangular selection accepting events with two correctly reconstructed $D$ quarks. The $Z j$ and $W j$ acceptance widths for different mass values are given in Tables 3 and Table 4 . Here the selection for type B events of $m_{D}=$ $600 \mathrm{GeV}$ is shown

quarks obtained from $Z$ and $W$ in the same event. The $D$ quark invariant mass reconstruction spectra obtained with this method are shown in Fig. 8 for case A, where $Z$ and $W$ decay modes involve leptons of the same generation and in Fig. 9 for case B, where $Z$ and $W$ decay modes involve leptons of different generations. The figures contain, for an integrated luminosity of $100 \mathrm{fb}^{-1}$ the reconstructed invariant mass distributions of $D \rightarrow Z j$ and $D \rightarrow W j$ processes. The latter have wider invariant mass spectra due to the use of the "visible mass" of the $W$ in the reconstruction routine.



Fig. 11. Multiplicity and $p_{\mathrm{T}}$ jets for signal ( white) and background (shaded) events for $2 \ell+4 j$ channel 
The number of signal $(S)$ and background $(B)$ events together with calculated statistical significance, corresponding to $100 \mathrm{fb}^{-1}$ integrated luminosity are presented in Tables 3 and 4 for type $A$ and $B$ events. The number of events in the signal and background processes were calculated in sliding mass windows of $\pm 2 \times \delta M$ around the peaks of the signal events for both $W j$ and $Z j$ invariant mass histograms. This selection aimed to select the events where both $D$ quarks were successfully reconstructed. The example of the sliding windows mass cuts to calculate the number of events in signal and the background is presented in Fig. 10 for type B events and for a $D$ quark mass of $600 \mathrm{GeV}$. The selection windows for all $D$ quark mass values are given in Tables 3 and 4 for type A and B events. The same tables also contain the statistical significance of the signal calculated as $S / \sqrt{B}$ if the total number of events were larger than 25 . Otherwise, the signal observation probability was calculated using Poisson statistics and the significance of a normalized Gaussian distribution yielding the same probability was reported.

\subsection{Search using the $2 \ell+4 j$ channel}

This channel has one $D$ quark decaying through a $Z$ boson which is expected to be reconstructed from two leptons (electrons and muons) and a second $D$ quark decaying either via a $W$ boson or a second $Z$ boson which can also be reconstructed using two energetic jets. One should note that the reconstruction efficiency of a highly boosted vector boson decaying hadronically can be lower compared to smaller boosts since the small opening angle between the jets can lead to a single jet. The signal events were simulated for both the $Z W j j$ and $Z Z j j$ cases. For this type of process, the signal is sought in a final state made of a pair of energetic electrons (or muons) in a multijet environment which includes two high $p_{\mathrm{T}}$ jets. The background events consisted of the SM $W Z+2 j, Z Z+2 j$ and $Z+2 j$ processes, with generator level cross sections of $6.19,5.5$ and $25 \mathrm{pb}$ respectively with the generator level selection requirements in set (2) except $p_{\mathrm{T}, p}>50 \mathrm{GeV}$.

The transverse momentum distribution of the leptons in the final state considered in this section do not differ much from those presented in the previous section. Therefore, Fig. 11 contains only the multiplicity of jets and their transverse momentum spectra for signal events using various $D$ quark mass in comparison with the corresponding spectra from background events. As it can be seen in the same figure, the signal process produces more energetic jets in the final state as compared to the SM background. However the jet multiplicity is higher than the case considered in the previous section. In general, high jet multiplicity creates problems in the invariant mass reconstruction due to combinatorics.

The cuts for the reconstruction of the pair produced $D$ quarks with mass of $600 \mathrm{GeV}$ are presented in the set below:

$$
\begin{array}{r}
\# \ell(e, \mu)=2, \\
\# \text { jet } \geq 4,
\end{array}
$$
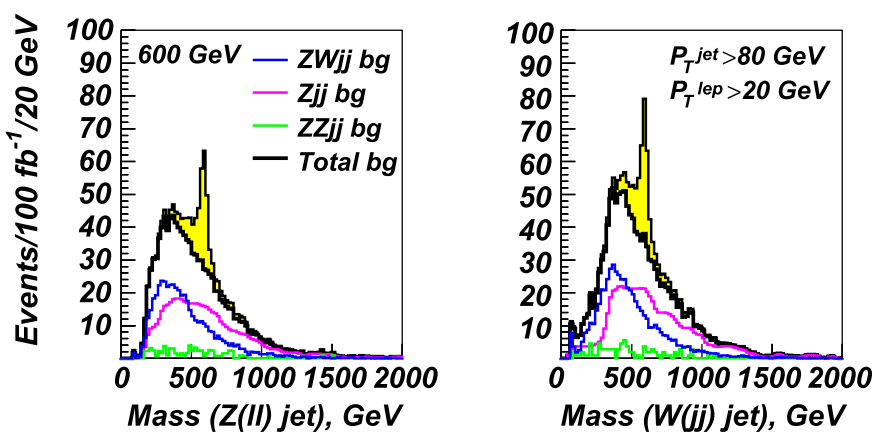

(a) $Z W j j$ with $m_{D}=600 \mathrm{GeV}$
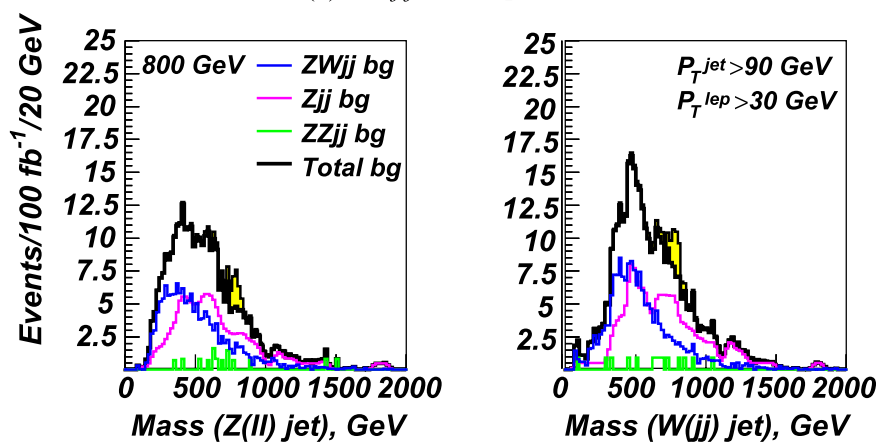

(b) $Z W j j$ with $m_{D}=800 \mathrm{GeV}$
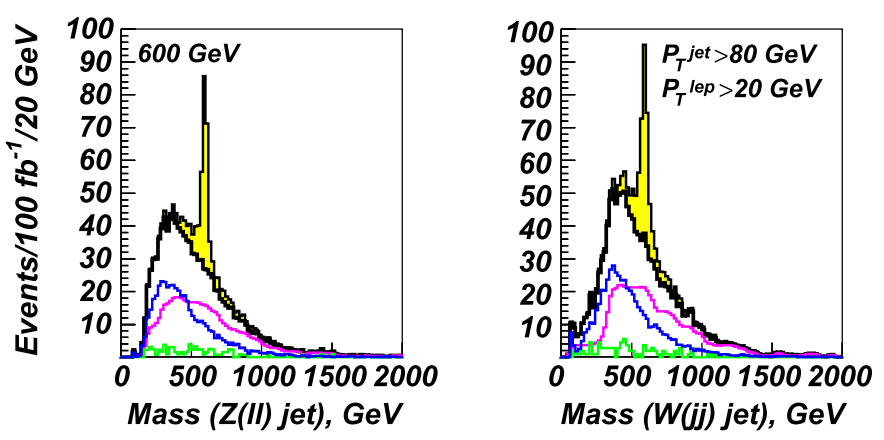

(c) $Z W j j$ with $m_{D}=600 \mathrm{GeV}$
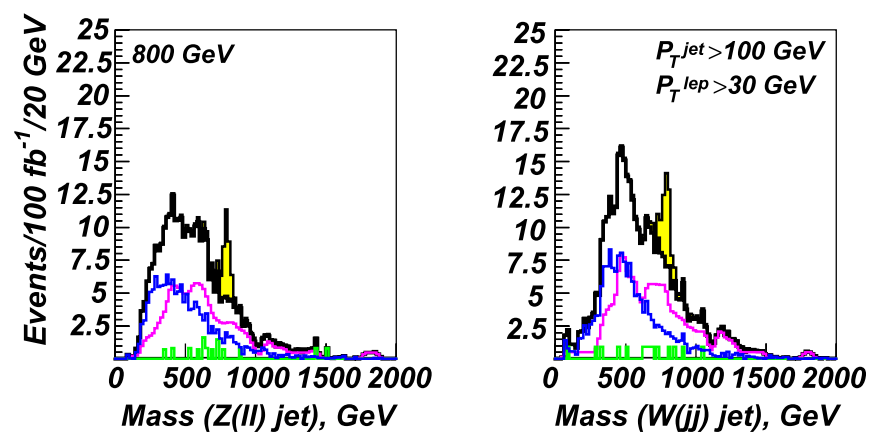

(d) $Z W j j$ with $m_{D}=800 \mathrm{GeV}$

Fig. 12. Invariant mass reconstruction spectra of signal (shaded) and backgrounds for $2 \ell+4 j$ channel

$$
\begin{aligned}
p_{\mathrm{T}, j} & >80 \mathrm{GeV}, \\
p_{\mathrm{T}, \ell} & >20 \mathrm{GeV}, \\
|\eta(j, \ell)| & <2.5 \\
M_{\ell \ell}^{\mathrm{rec}} & =90 \pm 20 \mathrm{GeV}, \\
M_{j j}^{\mathrm{rec}} & =85 \pm 25 \mathrm{GeV} .
\end{aligned}
$$


For higher $D$ quark mass values of 800 and $1000 \mathrm{GeV}$, the jet transverse momentum cuts were increased by 10 and $20 \mathrm{GeV}$ respectively for each next mass value to obtain a better value for signal significance $(S / \sqrt{B}$ or from Poisson statistics, depending on the available number of events as mentioned before), wherever it was statistically possible. The reconstruction of two lepton invariant mass was performed to obtain the mass of $Z$ boson.

The $D$ quark invariant mass reconstruction spectra with transverse momentum cuts applied are shown in Fig. 12. The mass of $D$ quark, decaying to a $Z$ boson and a jet was reconstructed by combining two highest $p_{\mathrm{T}}$ electrons (or muons) with a leading, or a next to the leading $p_{\mathrm{T}}$ jet choosing the case with the minimum mass difference between the two $D$ quark candidates as in the previous subsection. The invariant mass spectra for $D \rightarrow Z+$ jet decays are shown on the left side of Fig. 12 for each considered mass. The decays of a $D$ quark to $W+j e t$ were obtained by requiring the reconstruction of $W$ mass with two jets in the $(80 \pm 20) \mathrm{GeV}$ window and then by combination of the two " $W$-tagged" jets with the leading or next to leading $p_{\mathrm{T}}$ jet. The invariant mass spectra of $D \rightarrow W+$ jet decays are presented on the right side of the Fig. 12 for each considered mass. The distributions are shown for an integrated luminosity of $100 \mathrm{fb}^{-1}$.

The sum of the number of signal and background events from both $W$ and $Z$ involving cases with calculated statistical significance, corresponding to one LHC year at high luminosity is presented in Table 5 . Numbers of events in the signal and background events were calculated in the mass window of $\pm 2 \times \delta M$ around the respective mass peak in the signal events. Since this decay channel has the biggest branching ratio among all $D$ quark pair production processes, despite the obvious challenge of multi-jet environment, the statistical significance seems sufficient to observe $D$ quarks up to a mass of around $800 \mathrm{GeV}$.

Table 5. The signal and background cross sections before and after the event selection (B.S. and A.S.) with the relevant decay branching ratios folded in. The expected number of signal $(S)$ and background $(B)$ events in the mass window around the peak and the statistical significance for the $2 \ell+4 j$ channel, after the cuts given in equation set (7), for $100 \mathrm{fb}^{-1}$ of integrated luminosity

\begin{tabular}{lccc}
\hline Mass $(\mathrm{GeV})$ & 600 & 800 & 1000 \\
\hline$\sigma_{\text {signal B.S. }(\mathrm{fb})}$ & 20.1 & 3.82 & 0.74 \\
$\sigma_{\text {signal }}$ A.S. (fb) & 2.23 & 0.32 & 0.08 \\
$\sigma_{Z W j j}$ B.S. (fb) & 286.16 & 286.16 & 286.16 \\
$\sigma_{Z W j j}$ A.S. (fb) & 5.24 & 3.26 & 0.18 \\
$\sigma_{Z Z j j}$ B.S. $(\mathrm{fb})$ & 258.0 & 258.0 & 258.0 \\
$\sigma_{Z Z j j}$ A.S. $(\mathrm{fb})$ & 10.28 & 3.02 & 0.52 \\
$\sigma_{Z j j}$ B.S. $(\mathrm{fb})$ & 1700 & 1700 & 1700 \\
$\sigma_{Z j j}$ A.S. $(\mathrm{fb})$ & 13.58 & 8.36 & 0.53 \\
$\delta M(D \rightarrow Z / W$ jet$)(\mathrm{GeV})$ & 120 & 140 & 160 \\
$S$ & 133 & 18 & 2.5 \\
$B$ & 9 & 3 & 2.8 \\
significance & 44.3 & 6.8 & 1.7 \\
\hline
\end{tabular}

\section{Results and conclusions}

The analysis presented here has shown that the lightest of the quarks predicted by the $E_{6}$ GUT models can be discovered in different decay channels if its mass is less than $1 \mathrm{TeV}$. By combining multiple channels from this study and from the previous one [16], the discovery potential becomes higher and consequently the required luminosity becomes lower. Table 6 shows the expected number of signal and background events from each channel and the total significance after one year of running at nominal LHC luminosity of $100 \mathrm{fb}^{-1}$ per year. To combine the significance of the different channels, we have used a simple method. For each channel, after obtaining the probability of compatibility with a background hypothesis using Poisson or Gauss statistics depending on the available number of events, we calculated the total probability for background compatibility of all four considered channels. The total probability was converted to significance assuming a normalized Gauss distribution. The top plot in Fig. 13 shows the combined significance for 30 and $100 \mathrm{fb}^{-1}$ of integrated luminosity as a function of the $D$ quark mass. The same figure, on the bottom part, contains the required luminosity in $\mathrm{fb}^{-1}$ to observe $3 \sigma$ and $5 \sigma$ signals also as a function of the $D$ quark mass. The required luminosity was calculated assuming Poisson statistics and the total number of events were rounded down to the nearest integer. The presence of at least one event was required for any given channel to be included in the total significance calculation.

In conclusion, we show that the combination of the studied channels allows the discovery of the lightest isosinglet quark predicted by the $E_{6}$ GUT model within one year of LHC running time if its mass is up to about $950 \mathrm{GeV}$ with a significance of $5 \sigma$ or more. As pair production was considered, these results are independent of the mixing angle between the $D$ quark and its SM counterparts pro-

Table 6. Signal and background events for different channels and different masses of the $D$-quark for $100 \mathrm{fb}^{-1}$ integrated luminosity. Combined total significance is also given as the last row

\begin{tabular}{lccl}
\hline$m_{D}(\mathrm{GeV})$ & 600 & 800 & 1000 \\
\hline $4 \ell+2 j$ signal & 16 & 3.7 & 0.74 \\
background & 3.0 & 1.3 & 0.4 \\
$-\ln \mathrm{p}$ & 21.47 & 4.78 & 1.44 \\
$2 \ell+2 j+\#_{T}$ signal & 53 & 19 & 4 \\
background & 12 & 13 & 5 \\
$-\ln \mathrm{p}$ & 120 & 15.81 & 3.32 \\
$3 \ell+2 j+E_{T}$ signal & 97 & 18.3 & 1.8 \\
background & 24.9 & 9.0 & 1.05 \\
$-\ln \mathrm{p}$ & 191.4 & 20.66 & 1.69 \\
$2 \ell+4 j$ signal & 133 & 18 & 2.5 \\
background & 9 & 3 & 2.8 \\
$-\ln \mathrm{p}$ & 983 & 25.3 & 2.44 \\
$-\Sigma \ln \mathrm{p}$ & 1315.9 & 66.5 & 8.9 \\
combined significance $(\sigma)$ & 51.3 & 11.3 & 3.8 \\
\hline
\end{tabular}





Fig. 13. Upper plot: Combined significance as a function of the $D$ quark mass for an integral luminosity of 30 (dashed line) and 100 (solid line) $\mathrm{fb}^{-1}$. Lower plot: Required luminosities for the $3 \sigma$ observation limit (dashed line) and the $5 \sigma$ discovery limit as a function of the $D$ quark mass

vided that the lightest isosinglet quark mixes mainly to the first or second SM families. Although this study is based on a fast simulation of the detector response which was not fully validated and there are uncertainties associated with the QCD scale, statistical errors etc, we believe that the conservative selection cuts and the simplicity of the reconstruction algorithms give reliability to the conclusions. Additionally, the verification of these results using the full simulation of the detector is underway as preparation to first data from the LHC [19].

Acknowledgements. The authors would like to thank L. Tremblet and CERN Micro Club for kindly providing computational facilities, F. Ledroit, G. Azuelos and G. Brooijmans of ATLAS experiment for useful discussions. R.M. would like to thank A. Belyaev for his assistance in model calculations. R.M. also thanks NSERC/Canada for their support. A.S.'s work has been supported by a Marie Curie Early Stage Research Training Fellowship of the European Community's Sixth Framework Programme under contract number (MEST-CT-2005-020238). S.S acknowledges the support from the Turkish State Planning Committee under the contract DPT2002K-120250. G.U.'s work is supported in part by U.S. Department of Energy Grant DE FG0291ER40679. This work has been performed within the ATLAS Collaboration with the help of the simulation framework and tools which are the results of the collaboration-wide efforts.

\section{References}

1. F. Gursey, P. Ramond, P. Sikivie, Phys. Lett. B 60, 177 (1976)

2. F. Gursey, M. Serdaroglu, Lett. Nuovo Cimento 21, 28 (1978)

3. J. Hewett, T. Rizzo, Phys. Rep. 183, 195 (1989)

4. Particle Data Group, W.-M. Yao et al., J. Phys. G 33, 1 (2006)

5. O. Cakir, M. Yilmaz, Europhys. Lett. 38, 13 (1997)

6. Y. Hosotani, Phys. Lett. B 126, 309 (1983)

7. B. McInnes, J. Math. Phys. 31, 2094 (1990)

8. S. Sultansoy, G. Unel, atl-phys-pub-2007-007, [hep-ex/ 0610064]

9. T.C. Andre, C.L. Rosner, Phys. Rev. D 69, 035009 (2004)

10. A. Pukhov, hep-ph/0412191

11. CompHEP Collaboration, E. Boos et al., Nucl. Instrum. Methods A 534, 250 (2004)

12. T. Stelzer, W.F. Long, Phys. Commun. 81, 357 (1994)

13. J. Pumplin, D.R. Stump, J. Huston, H.L. Lai, P. Nadolsky, W.K. Tung, JHEP 0207, 012 (2002) [hep-ph/0201195]

14. S. Sultansoy, G. Unel, M. Yilmaz, hep-ex/0608041

15. ATLAS Detector and Physics Performance Technical Design Report, CERN/LHCC/99-14/15 (1999)

16. R. Mehdiyev et al., Eur. Phys. J. C 49, 13 (2007)

17. T. Sjöstrand et al., Comput. Phys. Commun. 135, 238 (2001) (LU TP 00-30, [hep-ph/0010017])

18. E. Richter-Was et al., ATLAS Note PHYS-98-131(1998), http://www.hep.ucl.ac.uk/atlas/atlfast/

19. ATLAS di-leptons plus jets working group, ATLAS CSC note, in preparation 